\title{
Fullerenes as Polyradicals
}

\author{
Elena F.Sheka \\ Peoples' Friendship University of Russia, 117923 Moscow, Russia \\ sheka@icp.ac.ru
}

\begin{abstract}
Electronic structure of $\mathrm{X}_{60}$ molecules $(\mathrm{X}=\mathrm{C}, \mathrm{Si})$ is considered in terms of 60 odd electrons and spin-dependent interaction between them. Conditions for the electrons to be excluded from the covalent pairing are discussed. A computational spin-polarized quantumchemical scheme is suggested to evaluate four parameters (energy of radicalization, exchange integral, atom spin density, and squared spin) to characterize the effect quantitatively. A polyradical character of the species, weak for $\mathrm{C}_{60}$ and strong for $\mathrm{Si}_{60}$, is established.
\end{abstract}

Key words: fullerenes $\mathrm{X}_{60}(\mathrm{X}=\mathrm{C}, \mathrm{Si})$, polyradicals, quantum chemistry

\section{Introduction}

It cannot be said that fullerenes suffer from the lack of theoretical considerations. Both a basic molecule $\mathrm{C}_{60}$ and its homologues $\mathrm{C}_{70}, \mathrm{C}_{84}$, etc. as well as analogues $\mathrm{Si}_{60}, \mathrm{Ge}_{60}$ have been repeatedly and thoroughly studied [see 1-7 and references therein]. In some sense, the molecule turned out to be a proving ground for testing different computational techniques, from a simplest to the most sophisticated. Constantly justifying the molecule stability, steadily repeated attempts of the molecule calculations are concentrated mainly on the reliability of reproducing the molecule structure and its possible distortion. There have been no doubts therewith concerning covalent bonding of atoms in the molecules. It has been taken for granted that all valence electrons participate in covalent pairing. That was the reason for the closed shell approximation to be exploited independently of whichever computational method has been used. The first breakdown of the assurance of the approach validity has been made by a comparative examination of the $\mathrm{C}_{60}$ and $\mathrm{Si}_{60}$ molecules [8-10] that has shown a strange feature in the high-spin states behavior of the molecules. As occurred, a sequence of spinvarying states, singlet (RHF)-triplet-quintet formed a progressively growing series by energy for the $\mathrm{C}_{60}$ molecule while for the $\mathrm{Si}_{60}$ molecule energy of the triplet and quintet states turned out to drop drastically with respect to the RHF singlet. Obviously, the peculiarity has clearly demonstrated the difference in the electronic structure of both molecules. However, as 
occurred, the observation is of much bigger importance since it concerns the basic properties of odd electrons behavior in fullerenic structures. The current paper is devoted to the phenomenon which is based on the extraction of odd electrons from covalent coupling. The paper is arranged in the following way. Section 2 is devoted to conceptual grounds of the carried computational experiment. Section 3 is devoted to exchange integrals as the main energetic characteristics of the electron coupling. Section 4 presents the results for lone pairs of odd electrons a well as for a set of pairs incorporated in the $\mathrm{C}_{60}$ and $\mathrm{Si}_{60}$ structures. The essentials of the study are discussed in Section 5.

\section{Conceptual Grounds}

Fullerenes are typical species with odd electrons that is why a concept on aromaticity has been expanded over the species since the very moment of their discovery [11]. However further examinations have highlighted that in spite of extreme conjugation, fullerenes behave chemically and physically as electron-deficient alkenes rather than electron-rich aromatic systems $[12,13]$ so that the electrons pairing seems to be the main dominant of electronic structure. Conceptually, the problem of an electron pair is tightly connected with a fundamental problem of quantum theory related to the hydrogen molecule. According to the Heitler-London theory [14], two hydrogen atoms (electrons) retain their individuality (atomic orbitals, involving spin), and look like two individual radicals with spin $S=1 / 2$ when they are far from each other (weak interaction). When the distance approaches the interatomic chemical bond (strong interaction), the electrons, as well as their spins, become delocalized over both atoms, their properties are described by a generalized molecular function (molecular orbital) and spins are aligned in an antiparallel way to provide tight covalent bonding between the atoms. As shown by forthcoming calculations [15], a continuous transition in the electron behavior from free radical-like to tightly coupled covalent bonding is observed indeed when the distance between the atoms changes from the infinity to the chemical bond length. By other words, the covalent bonding fades away when the electron interaction is weakening.

In the consequence of the topic of the current papers, two problems should be pointed out when this fundamental finding occurred to be of crucial importance. The first concerns diradicals in organic chemistry [16-23]. The phenomenon is caused by a pair of odd electrons connected with either C-C or C-N and N-N atom pairs and is common for species largely varying by composition. Generalizing its main aspects, the phenomenon essentials are caused 
by a violation of the above-mentioned atomic coupling from the covalent one in the part connected with odd electrons. Scheme in Figure 1 explains the main points of the diradical problem. Initially doubly degenerated atomic levels $\Psi_{A}$, and $\Psi_{B}$ are splitted due to electron interaction with the energy difference $\Delta \varepsilon$. Two spins of the relevant electrons can be distributed over the splitted levels by five different ways. Configurations I, II, III, and IV are related to singlet state while the only configuration $\mathrm{V}$ describes the triplet one. The latter is identical to that one with both spins directed down. As a result, the triplet state is spin-pure at any $\Delta \varepsilon$, while the singlet state is either purely covalent (configuration I) and, consequently, spin-pure at large $\Delta \varepsilon$, or is a mixture of configurations I-IV and becomes spin-mixed at lower $\Delta \varepsilon$. The energy difference $\Delta \varepsilon$ turns out to be the main criterion for attributing the species to either covalently bound or diradical species and the analysis of carbenes [19,23] can be considered as the best example of this kind.

The other problem is related to molecular magnets presented by dimers composed of two transition metal atoms surrounded by extended molecular ligands [24-26]. Two odd electrons

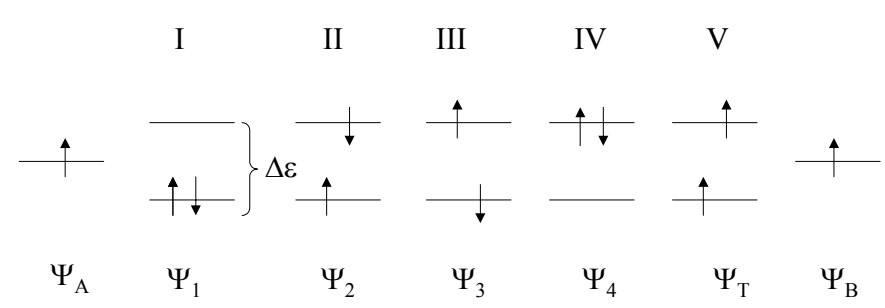

Figure 1. Diagram of the energy level and spin configurations of a lone electron pair

are associated with the metal atoms and their interaction is a priori weak. As previously, the triplet state is spin-pure while the singlet state is spin-mixed and is described by a combination of functions $\Psi_{1}, \Psi_{2}$, and $\Psi_{3}$ [27]. First attempts of the electron interaction analysis have been based on the direct consideration of configuration interaction $[24,25]$. However, as pointed in [26], it is more natural to consider the electron interaction within the SCF approximation by using different MOs for electrons with different spins [28-30]. The method is well equivalent to that involving configuration interaction that was exemplified in the case of conjugated molecules [30]. Later on Noodleman [31] explicitly elaborated the technique, called as broken symmetry approach [32], making it a practically feasible computational scheme based on quantum-chemical spin-polarized technique. 
Following these general concepts, two fullerenes $\mathrm{C}_{60}$ and $\mathrm{Si}_{60}$ have been considered in the current study. The analysis has been done in due course of extended computational experiment fulfilled in the framework of spin-polarised Hartree-Fock calculations that has highlighted the main characteristics, which are responsible for the molecule peculiar behavior.

\section{Exchange Integrals}

As shown for both diradicals [19,20] and molecular magnets [25], the criterion based on the quantity $\Delta \varepsilon$ lays the foundation of a qualitative analysis of the phenomenon, whilst important when tracing the odd electrons behavior when changing, say, structural parameters of the species. At the same time, as shown in the previous Section, the peculiarities of the odd electron pair behavior are caused by spin-mixing related to the singlet state of the pair. Therefore, the spin-dependent energy should be more appropriate quantitative characteristic of the phenomenon. Actually, as mentioned earlier, the value gradually decreases when weakening the electron interaction as shown for the hydrogen molecule [15]. In its turn, decreasing the value under controlled conditions will indicate growing the deviation of the electron coupling from the covalent bonding. Therefore, the problem is concentrated now around correct estimation of the value.

Let us consider two limit cases of strong and weak electron interaction. Obviously, diatomic molecules cover the former case. According to the Heitler-London theory [14], the energy of states of spin multiplicity $2 S+1$, where $S$ is full spin of two atoms with spins $S_{A}$ and $S_{B}$ each, ranging from 0 to $S_{A}+S_{B}$, can be expressed as

$$
E^{2 S+1}=E(0)-4 S_{A} S_{B} J_{A B} \sim E^{\prime}(0)+S(S-1) J_{A B} .
$$

Here $E(0)$ and/or $E^{\prime}(0)$ is the energy of the singlet state formed by covalently coupled electron pairs, $S_{A}$ and $S_{B}$ denote remained free spin of atoms $A$ and $B$, integral $J_{A B}$ describes

the electron exchange. In case of homonuclear molecules, $S_{A}^{\max }=S_{B}^{\max }=n / 2$, where $n$ determines the number of unpaired electrons. The expression was inspired by the Heizenberg theory of ferromagnetism [33] and occurred to be quite useful practically in describing highspin states of diatomic molecules [14]. Shown, the exchange integral is negative for the majority of molecules with only rare exclusion such as oxygen molecule and a few others. Silently implied therewith, the high-spin states are spin-pure that explains the appearance of 
spin-dependent part in Ex.(1) in form $S(S-1) J_{A B}$ where factor $S(S-1)$ corresponds to the eigen value of operator $\hat{S^{2}}$. Applying to the general problem of odd electrons, Eq.(1) suggests the integral $J_{A B}$ to be the main energetic criterion of the electron behavior in the limit of strong interaction. In what follows, the expression will be in use in the form

$$
J=\frac{E^{2 S_{\max }+1}-E^{0}}{S_{\max }\left(S_{\max }-1\right)},
$$

where $E^{2 S_{\max }+1}$ and $E^{0}$ are related to the states of the highest and the lowest multiplicities, respectively. A practical usefulness of the expression is resulted from the fact that both needed energies can be quite accurately determined by using modern quantum-chemical tools. The value $E^{0}$, related to covalently bound singlet state, is well determined by a closed shell version of a selected technique while the $E^{2 S_{\max }+1}$ value, that corresponds to the ferromagnetic alignment of all spins, is given by an open shell version of the technique. The ferromagnetic spin configuration is unique under any conditions (see Figure 1) so that the relevant solution is always true and the corresponding eigenfunctions satisfy both the Hamiltonian and $\hat{S^{2}}$ operator equations. Below a Hartree-Fock technique will be used for the values determination so that Eq.(2) can be rewritten in the following way

$$
J=\frac{E^{U H F}\left(S_{\max }\right)-E^{R H F}(0)}{S_{\max }\left(S_{\max }-1\right)} .
$$

Oppositely to covalently bound unique one-determinant singlet state in the limit of strong interaction, the state becomes broken by both space and spin symmetry [34] when odd electron interaction weakens. As suggested in [31], the one-determinant singlet wave function in this case can be expressed as [35]

$$
\begin{aligned}
\left|\psi_{B}\right\rangle & =(N !)^{-1 / 2} M^{-1 / 2} \operatorname{det}\left[\left(a_{1}+c b_{1}\right) \alpha, a_{2} \alpha \ldots a_{n} \alpha \mid\left(b_{1}+c a_{1}\right) \beta, b_{2} \beta \ldots b_{n} \beta\right] \\
& \approx M^{-1 / 2}\left(\phi_{1}+c \phi_{2}+c \phi_{3}\right) .
\end{aligned}
$$

The principal determinant $\phi_{1}$ describes pure covalent coupling of $n$ odd electrons while small amounts of the charge transfer determinants $\phi_{2}$ and $\phi_{3}$, corresponding to $A^{-}-B^{+}$and $A^{+}-B^{-}$configurations (see II and III in Fig.1) are mixed in due to nonorthogonality of atomic orbitals $\overline{a_{1}}=a_{1}+c b_{1}$ and $\overline{b_{1}}=b_{1}+c a_{1}$. The open shell manner for the function $\left|\psi_{B}\right\rangle$ is 
just appropriate to distinguish electron spins of atoms $A$ and $B$. The function corresponds to the antiferromagnetic (AF) alignment of spins of odd electrons.

As shown in [31], the energy of the above AF state is a specific weighted average of the energies of the pure spin multiplets. On the other hand, according to Eq.(4) it can be expressed as

$E_{B} \equiv E_{A F}^{U H F}=E_{\mathrm{cov}}-E_{\text {rad }}$,

where the latter is originated from the ionic contributions and is an independent measure of the extraction of odd electrons from the covalent coupling. The term can be called as the energy of either radicalization, or spin-mixing, or non-covalence depending on which namely aspect is to be emphasized. In what follows the first nomination is preferred. Since both energies $E_{A F}^{U H F}$ and $E_{\text {cov }}=E_{A F}^{R H F}$ can be calculated within the same QCh approach by using the corresponding open shell and closed shell versions, the $E_{\text {rad }}$ energy can be readily evaluated as the difference $E_{r a d}=E_{A F}^{R H F}-E_{A F}^{U H F}$. Since ionic energies are always negative, $E_{\text {rad }} \geq 0$.

When odd electrons are covalently coupled, $E_{A F}^{U H F}=E_{A F}^{R H F}$ and, consequently, $E_{\text {rad }}=0$. The corresponding exchange integral $J$ which provides the high-spin series of the electron energies has to be determined by Eq.(3). In its turn, $E_{\text {rad }} \neq 0$ is an unambiguous indication that the odd electron coupling deviates from the covalent one. As suggested in [31], the $J$ values can be determined therewith according to the following expressions

$E_{A F}^{U H F}=E_{F}^{U H F}+S_{\max }^{2} J$

and

$J=\frac{E_{A F}^{U H F}-E_{F}^{U H F}}{S_{\max }^{2}}$

where $E_{A F}^{U H F}$ and $E_{F}^{U H F}$ correspond to the lowest $(S=0)$ and highest $\left(S=S_{\max }\right)$ multiplicity of the $n$ electron system and are determined by one of the spin-polarized UHF technique. As has been already mentioned, the ferromagnetic state always corresponds to a true solution 
of the relevant QCh equations. According to [31], energies of the series of high-spin-pure states are described as

$E(S)=E_{A F}(0)-\frac{S(S+1)}{S_{\max }^{2}}\left(E_{A F}^{U H F}-E_{F}^{U H F}\right)=E_{A F}(0)-S(S+1) J$

where pure singlet state has the form

$E_{A F}(0)=E_{F}^{U H F}+S_{\max }\left(S_{\max }+1\right) J=E_{A F}^{U H F}+S_{\max } J$

It is important to notice that Eqs. (3), (6)-(9) are valid not only for lone pair of odd electrons. They retain their form in the case of $n$ identical pairs with that difference that the exchange integral $J$ is substituted by $\sim J / n$. In the weak interaction limit it is followed from the explicit expressions for the integral [31]. In the limit of strong interaction it was proved by a comparative study of the $\mathrm{H}_{2}$ and $\mathrm{H}_{6}$ systems $[15,36]$.

Equations (3), (7) and (8) form the ground of the carried computational experiment which is aimed at analysis of the odd electron properties of two fullerene molecules $\mathrm{C}_{60}$ and $\mathrm{Si}_{60}$. The computations have been performed by using semiempirical spin-polarized CLUSTER-Z1 sequential codes [37] in the version which is adequate to the AM1 technique [38]. Additionally to the mentioned, two other quantities were calculated, namely, eigenvalues of the $\hat{S^{2}}$ operator $[29,39]$

$$
(S * * 2)^{U H F}=1 / 4\left(N_{\alpha}-N_{\beta}\right)^{2}+1 / 2\left|N_{\alpha}-N_{\beta}\right|-S p\left(P^{\alpha} S P^{\beta} S\right)
$$

and spin density at atom $A$

$$
D_{s p, A}=\sum_{i \in A} P_{i i}^{\alpha}-P_{i i}^{\beta} \text {. }
$$

Here $N_{\alpha}$ and $N_{\beta} \quad\left(N_{\alpha} \geq N_{\beta}, N_{\alpha}+N_{\beta}=N\right.$, $)$ are the numbers of electron with spin up and down, respectively, $N$ is the total number of electrons while $P^{\alpha}$ and $P^{\beta}$ present the relevant density matrices. A comparison of the $(S * * 2)^{U H F}$ values [40] with the exact $(S * * 2)=S(S-1)$ makes possible an analysis of the purity of the considered spin states [41]. 


\section{Results}

\subsection{One Electron Pair in the $\mathbf{X}_{60}$ Structure}

In both organic and silicon chemistry the atom composition of pairs with odd electrons is rather variable (see, for example, $[16,20]$ ). Below we shall restrict ourselves by pairs of the $>\mathrm{C}-\mathrm{C}<$ and $>\mathrm{Si}-\mathrm{Si}<$ (below $>\mathrm{X}-\mathrm{X}<$ ) type only, where each atom is connected with three neighbors and which are characteristic for fullerenes $\mathrm{X}_{60}$. Individual pairs in the fullerenes structure can be formed by a virtual dehydrogenation of the $\mathrm{X}_{60} \mathrm{H}_{60}$ molecules, as shown in Figure 2. Both basic molecules are tightly bonded covalently with $E_{r a d}=0$ (see Table 1). Similar hexagon fragments were selected within the molecule structure which were then partially dehydrogenated that resulted in the formation of 1,2- and 1,4- pairs of odd electrons. The calculated values $E^{R H F}, E_{A F}^{U H F}$, and $E_{F}^{U H F}\left(S_{\max }=1\right)$ are listed in Table 1 [44]. Hereinafter Eq.(3) was used when determining exchange parameter $J$ for pairs with $E_{r a d}=0$ while Eq.(7) was applied to determine $J$ for pairs with $E_{r a d}>0$.

According to Hoffman's classification [19], the first of the mentioned pairs is related to via space one while the other presents a via bond pair. As seen from the table, the formation of the 1,2-pair in the $\mathrm{C}_{60} \mathrm{H}_{58}$ molecule does not disturb the covalent bonding since, as previously, $E_{r a d}=0$ so that $E_{A F}^{U H F}$ and $E_{F}^{U H F}$ describe spin-pure states with spin density at atoms equal either to zero or to one in the singlet and triplet states, respectively.

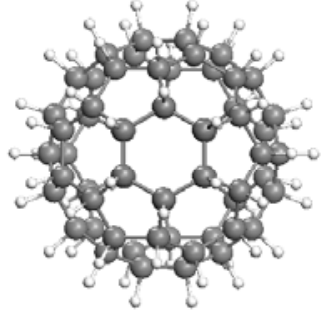

a

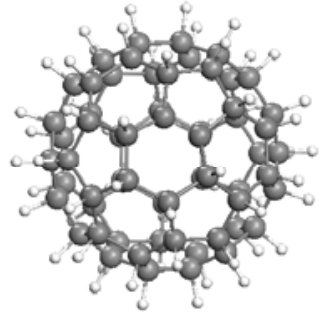

b

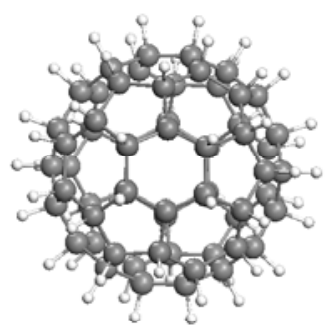

$\mathrm{C}$

Figure 2. Molecules $\mathrm{X}_{60} \mathrm{H}_{60}$ (a) and $\mathrm{X}_{60} \mathrm{H}_{58}$ with 1,2- (b) and 1,4- (c) pairs of odd electrons

Exchange parameter $J$ is rather big and similar to that one of the ethylene molecule (see Table 1). The other pair of the $\mathrm{C}_{60} \mathrm{H}_{58}$ molecule is characterized by a significant energy $E_{r a d}$, small exchange parameter $J$ and noticeable deviation of the calculated values $(S * * 2)^{U H F}$ from exact. Taking together, the features doubtlessly show the deviation from 
the covalent coupling in the pair that forces to take it as a diradical as conventionally accepted.

Oppositely to the carbon species, the formation of any pair in the $\mathrm{Si}_{60} \mathrm{H}_{58}$ molecule is followed by well evident diradical effects. Thus, energy $E_{\text {rad }}>0$ for both pairs; the values $(S * * 2)^{U H F}$ differ form the exact ones; atomic spin density $\mathrm{S}_{\mathrm{at}}$ at the pair atoms is large in the spin-mixed singlet state and considerably exceeds 1 in the triplet. As previously, the 1,2-pair and 1,4- pair differ rather drastically. The diradical character of both pairs is quite obvious. The discussed characteristics of the $\mathrm{Si}_{60} \mathrm{H}_{60}$ molecule pairs are similar to those of silicoethylene (see Table 1). As known [45], the latter does not exist in the gaseous state and is mentioned with respect to silicoethylene polymer that might be explained by its evidently diradical character.

\subsection{Set of Odd Electron Pairs in the $X_{60}$ Structures}

If lone odd electron pairs have been considered at least qualitatively and semiquantitatively $[19,20,23]$, the only study of a cyclic $\mathrm{H}_{6}$ cluster $[15,36]$ can be attributed to the examination of the pair sets. At the same time sets of pairs $>\mathrm{C}-\mathrm{C}<$ and $>\mathrm{Si}-\mathrm{Si}<$ are not a rarity for both organic and silicon chemistry. Enough to mention well extended class of aromatic compounds.

Since hexagon motive $\mathrm{X}_{6}$ is deeply inherent in fullerenic structures, its exploitation as a model set of odd electron pairs seems quite natural. Additionally, $\mathrm{X}_{10}$ configuration attracts attention since there are strong arguments to consider perdehydronaphthalene $\mathrm{C}_{10}$ as a building stone of the $\mathrm{C}_{60}$ molecule [46-48]. The corresponding two fragments studied in the current paper are shown in Figure 3 in the form of $\mathrm{X}_{60} \mathrm{H}_{54}$ and $\mathrm{X}_{60} \mathrm{H}_{50}$ molecules. As previously, those are formed by a virtual dehydrogenation of the basic $\mathrm{X}_{60} \mathrm{H}_{60}$ species. Two molecular species $\mathrm{X}_{6} \mathrm{H}_{6}$ and $\mathrm{X}_{10} \mathrm{H}_{8}$ are added to provide a completed picture of the pair sets. $\mathrm{X}_{60}$ molecules complete the study. The calculated characteristics are given in Tables 2 and 3.

$\mathbf{X}_{6} \mathbf{H}_{6}$ and $\mathbf{X}_{6}$ fragment. There are three electron pairs in the molecular structures, $S_{\max }=3$, and the relevant state of the ferromagnetic aligning of six spins corresponds to septet. As seen from Table 2, the $\mathrm{C}_{6} \mathrm{H}_{6}$ molecule is tightly bound covalently, $E_{\text {rad }}=0$. Both singlet and septet states are spin-pure, however, the singlet state spin density is slightly 

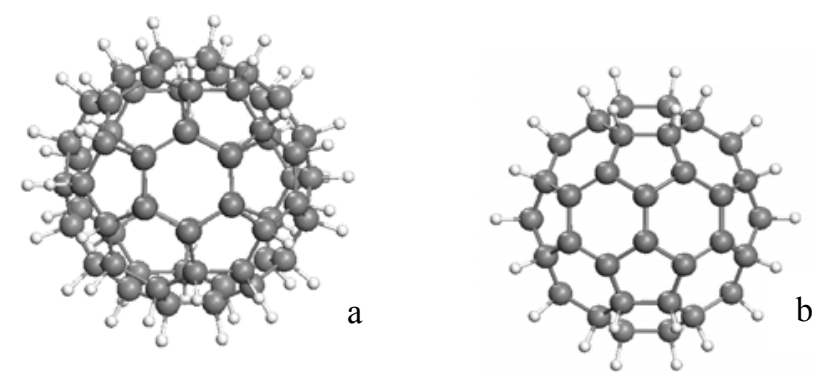

Figure 3. Molecules $\mathrm{X}_{60} \mathrm{H}_{54}$ (a) and $\mathrm{X}_{60} \mathrm{H}_{50}$ (b) with $\mathrm{X}_{6}$ and $\mathrm{X}_{10}$ fragments, respectively.

Table 1. Energetic characteristics of a lone pair of odd electrons in the $X_{60}$ structures $^{1}$

\begin{tabular}{|c|c|c|c|c|c|}
\hline $\begin{array}{l}\text { Molecular } \\
\text { species }\end{array}$ & Quantity & $R H F$ & $U H F(S=0)$ & $U H F\left(S_{\max }\right)$ & $E_{r a d}$ \\
\hline $\mathrm{C}_{60} \mathrm{H}_{60}$ & $\begin{array}{c}\Delta \mathrm{H}, \mathrm{kcal} / \mathrm{mol} \\
\left(S^{* * 2}\right)^{\text {UHF }}\end{array}$ & 334.161 & $\begin{array}{c}334.161 \\
0\end{array}$ & & 0 \\
\hline $\begin{array}{l}\mathbf{C}_{60} \mathbf{H}_{58} \\
\text { 1,2-pair }\end{array}$ & $\begin{array}{c}\mathrm{H}, \mathrm{kcal} / \mathrm{mol} \\
\left(S^{* *} 2\right)^{U H F} \\
D_{s p, A} \\
J, \mathrm{kcal} / \mathrm{mol}\end{array}$ & 316.319 & $\begin{array}{cc}316.315 & \\
0 & \\
0 / 0 & \\
& -1 \\
\end{array}$ & $\begin{array}{c}352.457 \\
2.021 \\
1 / 1 \\
07\end{array}$ & 0.004 \\
\hline $\begin{array}{l}\mathbf{C}_{60} \mathbf{H}_{58} \\
\text { 1,4-pair }\end{array}$ & $\begin{array}{c}\mathrm{H}, \mathrm{kcal} / \mathrm{mol} \\
\left(S^{* *} 2\right)^{U H F} \\
D_{s p, A} \\
J, \mathrm{kcal} / \mathrm{mol}\end{array}$ & 389.304 & $\begin{array}{c}334.422 \\
1.031 \\
-1.03 /+1.03 \\
-0\end{array}$ & $\begin{array}{c}334.623 \\
2.027 \\
+1.02 /+1.02 \\
\end{array}$ & 54.88 \\
\hline Ethylene & $\begin{array}{c}\mathrm{H}, \mathrm{kcal} / \mathrm{mol} \\
\left(S^{* *} 2\right)^{U H F} \\
D_{s p, A} \\
J, \mathrm{kcal} / \mathrm{mol}\end{array}$ & 16.449 & $\begin{array}{cc}16.449 & \\
0 & \\
0 / 0 & \\
& -1 \\
\end{array}$ & 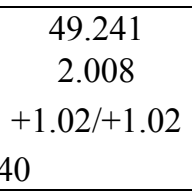 & 0 \\
\hline $\mathbf{S i}_{60} \mathbf{H}_{60}$ & $\begin{array}{c}\Delta \mathrm{H}, \mathrm{kcal} / \mathrm{mol} \\
\left(S^{* *}\right)^{\text {UHF }}\end{array}$ & 441.599 & $\begin{array}{c}441.597 \\
0\end{array}$ & & 0.002 \\
\hline $\begin{array}{l}\mathbf{S i}_{\mathbf{6 0}} \mathbf{H}_{\mathbf{5 8}} \\
\text { 1,2-pair }\end{array}$ & $\begin{array}{c}\Delta \mathrm{H}, \mathrm{kcal} / \mathrm{mol} \\
\left(S^{* *}\right)^{U H F} \\
D_{s p, A} \\
J, \mathrm{kcal} / \mathrm{mol}\end{array}$ & 461.070 & $\begin{array}{c}457.430 \\
1.050 \\
-1.5 /+1.5\end{array}$ & $\begin{array}{c}464.639 \\
2.148 \\
+1.31 /+1.31 \\
\end{array}$ & 3.64 \\
\hline $\begin{array}{l}\mathbf{S i}_{\mathbf{6 0}} \mathbf{H}_{58} \\
\text { 1,4-pair }\end{array}$ & $\begin{array}{c}\Delta \mathrm{H}, \mathrm{kcal} / \mathrm{mol} \\
\left(S^{* *}\right)^{U H F} \\
D_{s p, A} \\
J, \mathrm{kcal} / \mathrm{mol}\end{array}$ & 504.097 & $\begin{array}{r}453.606 \\
1.383 \\
-1.63 /+1.63 \\
-0 \\
\end{array}$ & $\begin{array}{c}454.510 \\
2.269 \\
+1.55 /+1.55 \\
04\end{array}$ & 50.49 \\
\hline Silicoethylene & $\begin{array}{c}\Delta \mathrm{H}, \mathrm{kcal} / \mathrm{mol} \\
\left(S^{* *} 2\right)^{U H F} \\
D_{s p, A} \\
J, \mathrm{kcal} / \mathrm{mol}\end{array}$ & 54.502 & $\begin{array}{c}48.934 \\
0.899 \\
-1.33 /+1.33 \\
-5\end{array}$ & $\begin{array}{c}54.185 \\
2.017 \\
+1.17 /+1.17 \\
\end{array}$ & 5.57 \\
\hline
\end{tabular}

${ }^{1}$ Ethylene and silicoethylene are calculated in the current study.

nonzero and is regularly distributed over the molecule atoms with values shown in the table. The exchange parameter $J$ is still big whilst lower with respect to that of ethylene 
molecule. The benzene fragment $\mathrm{C}_{6}$ of the $\mathrm{C}_{60} \mathrm{H}_{54}$ molecule behaves fully similarly so that its formation does not affect the covalent bonding. As in the case of the $\mathrm{C}_{6} \mathrm{H}_{6}$ molecule, singlet state spin density on the benzene fragment atoms is also nonzero and bigger than previously.

Absolutely another picture (see Table 3 ) can be seen for the $\mathrm{Si}_{6} \mathrm{H}_{6}$ molecule while both the molecule itself and its $\mathrm{Si}_{6}$ analogue in the $\mathrm{Si}_{60} \mathrm{H}_{54}$ species behave quite similarly. Those are characterized by large values of $E_{\text {rad }}$, by small parameter $J$ and by atomic spin densities, similar in value in both singlet and septet state. The latter is spin-pure enough while the singlet state is evidently spin-mixed since the obtained $(S * * 2)^{U H F}$ value differs significantly from zero. The discussed features force to admit that both silicobenzene molecule and its analogue in the $\mathrm{Si}_{60} \mathrm{H}_{54}$ molecule should be attributed to polyradicals.

$\mathbf{X}_{10} \mathbf{H}_{8}$ and $\mathbf{X}_{10}$ fragment. There are five odd electron pairs in the molecular species, $S_{\max }=5$, and the multiplicity of the ferromagnetic state is 11. Analyzing data given in Table 2, an unexpected discovery can be made concerning the violation of the molecule covalent bonding in the naphthalene $\mathrm{C}_{10} \mathrm{H}_{8}$. As far as known, the stability of the covalent bound singlet state of the molecule has never been in doubt. However, the carried calculations show that not only $E_{\text {rad }}$ noticeably differs from zero but $(S * * 2)^{U H F}$ is non-zero as well showing spin-mixed character of the singlet state. High values of atomic spin density are also remarkable. The findings evidence convincingly a polyradical behavior of the molecule, though not too strong As seen from Table 2, the tendency is not only kept but even strengthened for the $\mathrm{C}_{10}$ fragment in the $\mathrm{C}_{60} \mathrm{H}_{50}$ molecule. Supposing the fragment to be a building stone of the $\mathrm{C}_{60}$ molecule [39-41], its properties may genetically forecast a possible polyradical character of $\mathrm{C}_{60}$ [49].

The data in Table 3 related to siliconathtalene and $\mathrm{Si}_{10}$ fragment of the $\mathrm{Si}_{60} \mathrm{H}_{50}$ molecule leave no doubts concerning polyradical character of both molecules. Evidently, the effect is much bigger comparing with that of carbon species. For both silicon species $E_{\text {rad }}$ are big, $(S * * 2)^{U H F}$ drastically differs from zero for singlet states and even in the ferromagnetic states the $(S * * 2)^{U H F}$ values do not coincide with the exact ones. The latter is followed by a non-regular distribution of the atomic spin density over atoms. 
Table 2. Energetic characteristics of sets of odd electrons pairs in the $\mathrm{C}_{60}$ structures $^{1}$

\begin{tabular}{|c|c|c|c|c|c|}
\hline $\begin{array}{l}\text { Molecular } \\
\text { species }\end{array}$ & Quantity & $R H F$ & $U H F(S=0)$ & $U H F\left(S_{\max }\right)$ & $E_{r a d}$ \\
\hline \multirow[t]{4}{*}{$\mathrm{C}_{6} \mathrm{H}_{6}$} & $\Delta \mathrm{H}, \mathrm{kcal} / \mathrm{mol}$ & 21.954 & 21.952 & 162.350 & 0.002 \\
\hline & $(S * * 2)^{U H F}$ & 0 & 0 & 12.016 & \\
\hline & $D_{s p, A}$ & 0 & $-0.15 /+0.15$ & 1.01 & \\
\hline & $J, \mathrm{kcal} / \mathrm{mol}$ & \multicolumn{4}{|c|}{-11.70} \\
\hline \multirow{4}{*}{$\begin{array}{c}\mathrm{C}_{6} \\
\text { fragment } \\
\text { in } \mathrm{C}_{60} \mathrm{H}_{54}\end{array}$} & $\Delta \mathrm{H}, \mathrm{kcal} / \mathrm{mol}$ & 330.476 & 330.292 & 484.045 & 0.18 \\
\hline & $(S * * 2)^{U H F}$ & 0 & 0.207 & 12.027 & \\
\hline & $D_{s p, A}$ & 0 & $-0.29 /+0.29$ & 1.09 & \\
\hline & $J, \mathrm{kcal} / \mathrm{mol}$ & \multicolumn{4}{|c|}{-12.80} \\
\hline \multirow[t]{4}{*}{$\mathrm{C}_{10} \mathrm{H}_{8}$} & $\Delta \mathrm{H}, \mathrm{kcal} / \mathrm{mol}$ & 40.466 & 38.619 & 291.512 & 1.85 \\
\hline & $(S * * 2)^{U H F}$ & 0 & 0.743 & 30.025 & \\
\hline & $D_{s p, A}$ & 0 & $\begin{array}{l}-(0.41-0.47) / \\
+(0.41-0.47)\end{array}$ & $(0.97-1.09)$ & \\
\hline & $J, \mathrm{kcal} / \mathrm{mol}$ & \multicolumn{4}{|c|}{-10.12} \\
\hline \multirow{4}{*}{$\begin{array}{c}\mathrm{C}_{10} \\
\text { fragment } \\
\text { in } \mathrm{C}_{60} \mathrm{H}_{50}\end{array}$} & $\Delta \mathrm{H}, \mathrm{kcal} / \mathrm{mol}$ & 363.146 & 360.027 & 612.829 & 3.12 \\
\hline & $(S * * 2)^{U H F}$ & 0 & 1.009 & 30.035 & \\
\hline & $D_{s p, A}$ & 0 & $\begin{array}{l}-(0.50-0.52) / \\
+(0.50-0.52)\end{array}$ & $(0.96-0.97)$ & \\
\hline & $J, \mathrm{kcal} / \mathrm{mol}$ & \multicolumn{4}{|c|}{-10.11} \\
\hline \multirow[t]{4}{*}{$\mathrm{C}_{60}$} & $\Delta \mathrm{H}, \mathrm{kcal} / \mathrm{mol}$ & 972.697 & 955.380 & 2629.790 & 17.32 \\
\hline & $(S * * 2)^{U H F}$ & 0 & 4.937 & 930.386 & \\
\hline & $D_{s p, A}$ & 0 & $\pm(0.61-0)$ & $1.0-0.8$ & \\
\hline & $J, \mathrm{kcal} / \mathrm{mol}$ & \multicolumn{4}{|c|}{-1.86} \\
\hline
\end{tabular}

${ }^{1}$ Data dispersion is given in brackets

Fullerenes $\mathbf{C}_{60}$ and $\mathbf{S i}_{\mathbf{6 0}}$. There are 30 odd electron pairs in each molecule, $S_{\max }=30$, and the multiplicity of the states with ferromagnetic alignment of all 60 spins is 61 . As seen from Tables 2 and 3, $E_{\text {rad }}>0$ for both molecules, for the $\mathrm{Si}_{60}$ just drastically. The UHF singlet states are spin-mixed, and again, the mixing for silicon species is just enormous that is seen from the deviation of the $(S * * 2)^{U H F}$ values from zero. In both cases atomic spin density is quite considerable and is distributed over atoms in a rather complicated way. Figure 4 presents the spin density distribution in a manner when the presented value gradually grows from the left to the right while the sums over the values in both cases are zero. White-and-black image of the $\mathrm{X}_{60}$ molecule shown in the figure insert highlights the space distribution of positive and negative spin density over atoms. Taking together, the presented data make a polyradical character of the odd electron bonding in both molecules completely evident. 
Table 3. Energetic characteristics of sets of odd electrons pairs in the $\mathrm{Si}_{60}$ structures $^{1}$

\begin{tabular}{|c|c|c|c|c|c|}
\hline $\begin{array}{l}\text { Molecular } \\
\text { species }\end{array}$ & Quantity & $R H F$ & $U H F(S=0)$ & $U H F\left(S_{\max }\right)$ & $E_{\text {rad }}$ \\
\hline $\mathrm{Si}_{6} \mathrm{H}_{6}$ & $\begin{array}{l}\Delta \mathrm{H}, \mathrm{kcal} / \mathrm{mol} \\
\left(S^{* *} 2\right)^{U H F} \\
D_{s p, A} \\
J, \mathrm{kcal} / \mathrm{mol}\end{array}$ & $\begin{array}{c}144.509 \\
0 \\
0\end{array}$ & $\begin{array}{c}121.246 \\
2.678 \\
-1.51 /+1.51 \\
\end{array}$ & $\begin{array}{c}158.973 \\
12.029 \\
1.09\end{array}$ & 23.26 \\
\hline $\begin{array}{l}\mathbf{S i}_{6} \\
\text { fragment } \\
\text { in } \mathbf{S i}_{60} \mathbf{H}_{54}\end{array}$ & $\begin{array}{l}\Delta \mathrm{H}, \mathrm{kcal} / \mathrm{mol} \\
\left(S^{*} * 2\right)^{U H F} \\
D_{s p, A} \\
J, \mathrm{kcal} / \mathrm{mol}\end{array}$ & 511.168 & $\begin{array}{c}488.902 \\
3.174 \\
-1.67 /+1 / 67\end{array}$ & $\begin{array}{c}527.641 \\
12.164 \\
1.14-1.09\end{array}$ & 22.27 \\
\hline$\overline{\mathbf{S i}_{10} \mathbf{H}_{8}}$ & $\begin{array}{l}\mathrm{H}, \mathrm{kcal} / \mathrm{mol} \\
\left(S^{* * 2}\right)^{U H F} \\
D_{s p, A} \\
J, \mathrm{kcal} / \mathrm{mol}\end{array}$ & $\begin{array}{c}226.706 \\
0 \\
0\end{array}$ & $\begin{array}{c}188.134 \\
4.609 \\
-(1.53-1.71) / \\
+(1.53-1.71)\end{array}$ & $\begin{array}{c}242.668 \\
30.457 \\
\text { complicated } \\
\text { distribution }\end{array}$ & 38.57 \\
\hline $\begin{array}{l}\mathbf{S i}_{\mathbf{1 0}} \\
\text { fragment } \\
\text { in } \mathbf{S i}_{\mathbf{6 0}} \mathbf{H}_{\mathbf{5 0}}\end{array}$ & $\begin{array}{l}\Delta \mathrm{H}, \mathrm{kcal} / \mathrm{mol} \\
\left(S^{* * 2}\right)^{U H F} \\
D_{s p, A} \\
J, \mathrm{kcal} / \mathrm{mol}\end{array}$ & $\begin{array}{c}566.321 \\
0 \\
0\end{array}$ & $\begin{array}{c}519.85 \\
5.585 \\
-(1.68-1.93) / \\
+(1.68-1.93)\end{array}$ & $\begin{array}{c}599.43 \\
31.347 \\
\text { complicated } \\
\text { distribution }\end{array}$ & 46.47 \\
\hline $\mathbf{S i}_{60}$ & $\begin{array}{l}\Delta \mathrm{H}, \mathrm{kcal} / \mathrm{mol} \\
\left(S^{* * 2}\right)^{U H F} \\
D_{s p, A} \\
J, \mathrm{kcal} / \mathrm{mol}\end{array}$ & $\begin{array}{ll} & 1295.988 \\
0 & \\
0 & \end{array}$ & $\begin{array}{c}999.215 \\
31.764 \\
\pm(2.00-0.94)\end{array}$ & $\begin{array}{c}1513.208 \\
930.576 \\
(0.98-1.01)\end{array}$ & 296.77 \\
\hline
\end{tabular}

${ }^{1}$ Data dispersion is given in brackets

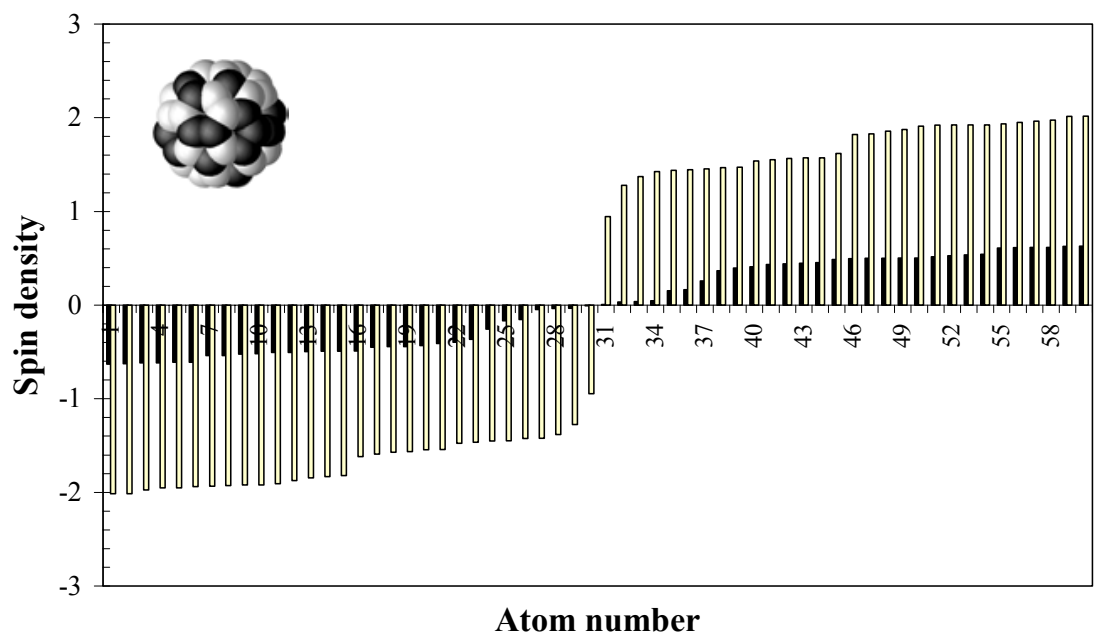

Figure 4. Spin density distribution over atoms of molecule $\mathrm{C}_{60}$ (dense black bars) and $\mathrm{Si}_{60}$ (contour bars) in the UHF singlet state. Insert: space distribution of the density for the $\mathrm{Si}_{60}$ molecule. 


\section{Discussion}

The carried analysis has convincingly shown that $\mathrm{X}_{60}$ fullerene composition of atoms with odd electrons results in weakening the covalent bonding that is just drastic in the case of silicon species. To describe the phenomenon concisely, the term polyradicalization has been suggested to emphasize that a rather peculiar chemical behavior should be expected from the species. As shown in the study, four parameters can be proposed to describe the effect quantitatively. The main parameter $E_{r a d}=E^{R H F}-E_{A F}^{U H F}$ indicates straightforwardly the bonding weakening, when it is non-zero. Three other parameters, namely, exchange parameter $J,(S * * 2)^{U H F}$, and $D_{s p, A}$, describe quantitatively conditions under which the weakening occurs. Taking together, they provide a complete picture of the phenomenon discussed. Additionally, the computational approach used makes it possible to determine the energy of high-spin states of the studied compounds. Figure 5 presents the data related to $E(S)$ and $(S * * 2)^{U H F}$ calculated in accordance with Eqs. (8) and (10) for both $\mathrm{C}_{60}$ and $\mathrm{Si}_{60}$ species. The series of the $E^{U H F}(S)$ energies of spin-mixed states, calculated straightforwardly by using the applied tool, are included for comparison. As seen from the figure, the difference between the series of spin-pure and spin-mixed states is not too big as might be expected. This is much more surprising since the calculated $E^{U H F}(S)$ relate to the optimized structures which are different for different spin states, sometimes rather significantly, while the $E(S)$ series is related to the same structure. A conclusion can be made that the applied spin-polarized QCh tool provides a quite reliable presentation of high-spin states. Curves 3 in the figure plots the ratio $\varsigma(\%)=\frac{(S * * 2)^{U H F}-S(S-1)}{S(S-1)}$ which characterizes spin purity of the states. As seen from the figure, in the case of $\mathrm{C}_{60}$, the highspin states become spin-pure at rather low spin values while only at high spins the similar is observed for the $\mathrm{Si}_{60}$ molecule. 

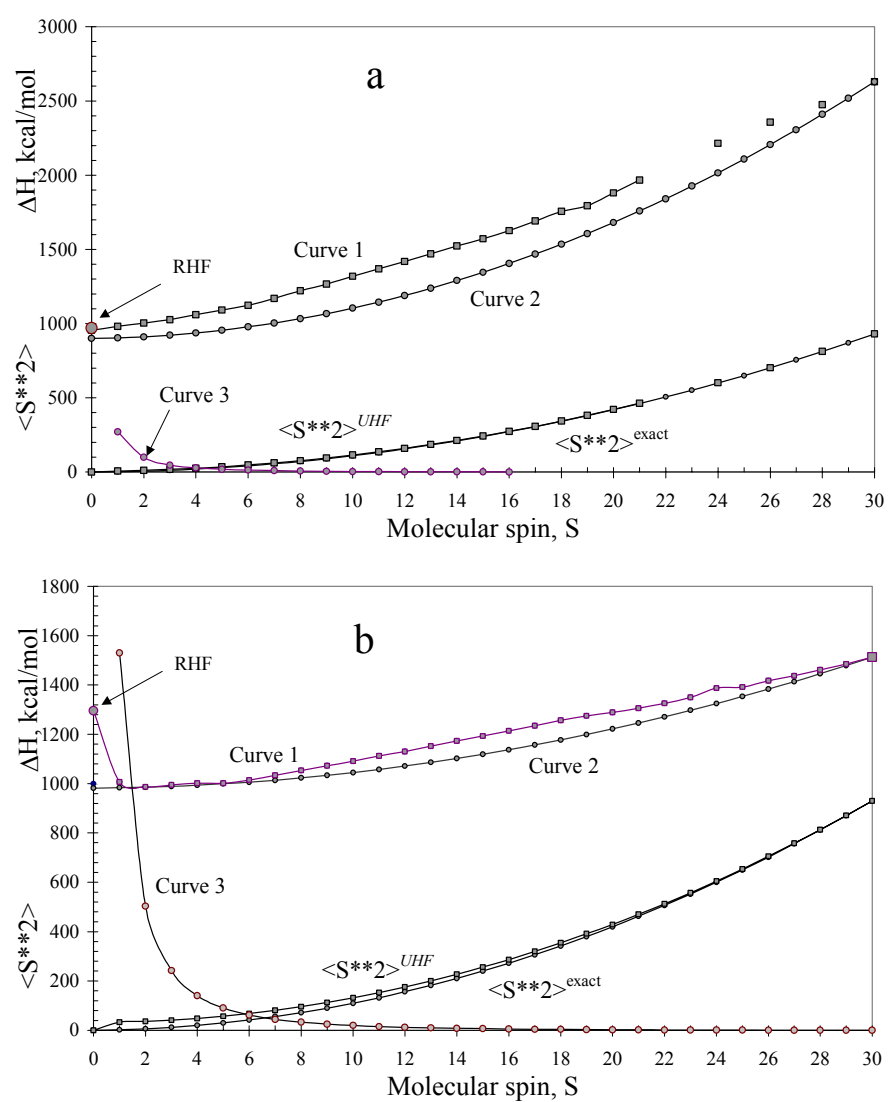

Figure 5. Heat of formation of the UHF- (curves 1) and pure- (curves 2) spin states of the molecules $\mathrm{C}_{60}$ (a) and $\mathrm{Si}_{60}$ (b); RHF singlet states are shown by arrows; curves 3 present $\zeta$ values (see text)

Even in the first studies of diradicals, Hoffman [17-19] and other authors [20] have tried to exhibit the criterion of the transition from covalent pairing to odd electron pair radicalization. However, only the energy difference $\Delta \varepsilon=\varepsilon_{2}-\varepsilon_{1}$ between the energies of two orbitals of the pair was suggested that was not enough to formalize the criterion. A considerable extension of the number of quantitative parameters, readily accessible by the modern spin-polarized QCh techniques, makes now possible to suggest a formal criterion for the transition. Given in Figure 6 presents the dependence of $E_{\text {rad }}$ versus exchange parameter $J$ on the basis of the data summarized in Table 4. As seen, the dependence for both carbon and silicon species is quite similar and exhibits a clearly seen quasi-threshold character. One may conclude that for the studied species the transition starts when $J$ reaches $\sim 10 \mathrm{kcal} / \mathrm{mol}$.

Dependencies $E_{\text {rad }}(J)$, or more precise, the steepness of the curves after transition, well formalize the difference in the polyradicalization of different species. As seen in the figure, the steepness is a few times more for the silicon species in comparison with carbon molecules. The obvious preference shown by silicon atoms towards polyradicalization 


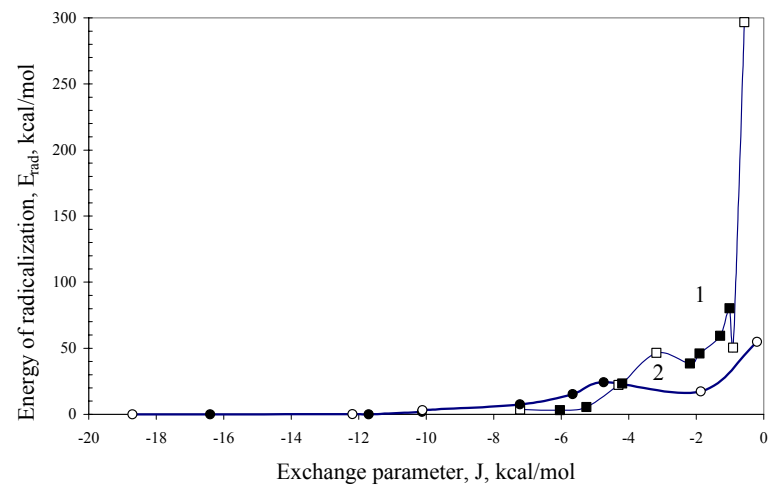

Figure 6. Energy of polyradicalization versus exchange parameter $J$ for odd electron pairs $>\mathrm{Si}-\mathrm{Si}<(1)$ and $>\mathrm{C}-\mathrm{C}<(2)$. Empty and filled points correspond to fullerenic and "aromatic" structures, respectively

instead of double bond formation is well supported by high values of atomic spin densities (see Tables 1 and 2). The latter quantity, in its turn, is provided by electrons taken out of chemical bonding [50]. Actually, Figure 7 presents absolute values of the atom spin density $\left|D_{s p, A}\right|$ multiplied by an electron spin, and atom free valence $V_{A}^{\text {free }}$ distributed over the molecules atoms. The latter is determined as

$V_{A}^{\text {free }}=N_{\text {val }}^{A}-\sum_{B \neq A} K_{A B}$

where $N_{v a l}^{A}$ is the number of valent electrons of atom $A$ while $\sum_{B \neq A} K_{A B}$ presents a generalized bond index [51], summarized over all atoms excluding atom $A$. A close similarity should be noted between the two values, which are calculated independently. Taking together, the data present a quantitative explanation of the difference in bonding carbon and silicon atoms, showing how much every odd electron is free of bonding. Therefore, silicon fullerene is, in average, of $\sim 100 \%$ polyradical while its carbon counterpart is only of $\sim 20 \%$ polyradical. This observation explains why silicon atoms "dislike" $s p_{2}$ hybridization $[9,10]$. On the other hand, this can be described as following. While carbon atom interaction forces odd electron to participate in the action thus strengthening it, silicon atoms prefer to leave the odd electrons free in a form of spin density, while the atom interaction is kept at much weaker level. As a result, the electronic structure of carbon atom occurs to be quite labile or soft while that of silicon atom is much 
Table 4. Fundamental energetic parameters of $>\mathrm{X}-\mathrm{X}<$ odd electron pairs, kcal $/ \mathrm{mol}$

\begin{tabular}{ccccc}
\hline \multirow{2}{*}{ Molecular species } & \multicolumn{2}{c}{ Carbon } & \multicolumn{2}{c}{ Silicon } \\
\cline { 2 - 5 } & $E_{\text {rad }}$ & $J$ & $E_{\text {rad }}$ & $J$ \\
\hline & & & & \\
$\mathrm{X}_{2} \mathrm{H}_{4}$ & 0.00 & -16.40 & 5.57 & -5.25 \\
1,2 pair $\mathrm{X}_{60} \mathrm{H}_{58}$ & 0.004 & -18.07 & 3.64 & -7.21 \\
1,4 pair $\mathrm{X}_{60} \mathrm{H}_{58}$ & 54.88 & -0.20 & 50.49 & -0.90 \\
$\mathrm{X}_{6} \mathrm{H}_{6}$ & 0.002 & -11.70 & 23.26 & -4.19 \\
$\mathrm{X}_{6}$ fragment of & 0.18 & -12.80 & 22.27 & -4.30 \\
$\mathrm{X}_{60} \mathrm{H}_{54}$ & & & & \\
$\mathrm{X}_{10} \mathrm{H}_{8}$ & 1.85 & -10.12 & 38.57 & -2.18 \\
$\mathrm{X}_{10}$ fragment of $_{\mathrm{X}_{60} \mathrm{H}_{50}}^{3.12}$ & -10.11 & 46.47 & -3.18 \\
$\mathrm{X}_{14} \mathrm{H}_{10}$ & 7.45 & -7.22 & 45.97 & -1.90 \\
$\mathrm{X}_{18} \mathrm{H}_{12}$ & 15.32 & -5.66 & 59.53 & -1.29 \\
$\mathrm{X}_{22} \mathrm{H}_{14}$ & 24.23 & -4.74 & 80.22 & -1.01 \\
$\mathrm{X}_{60}$ & 17.32 & -1.86 & 296.77 & -0.57 \\
\hline
\end{tabular}

more rigid. The difference could be naturally explained by the difference in the length of $\mathrm{C}-\mathrm{C}$ and $\mathrm{Si}-\mathrm{Si}$ bonds that differ by about $0.1 \mathrm{~nm}$. As shown by the example of 'via space' and 'via bond' lone C-C pairs in Section 3.1, changing the intrapair distance from 0.134 to $0.267 \mathrm{~nm}$ results in a drastic strengthening of the radical character of the pair, indeed. However, in case of silicon species, the difference is still much more so that some else silicon individual characteristic are important. The obtained findings throw light as well on why "...A comparison of the chemistry of tetravalent carbon and silicon reveals such gross differences that the pitfalls of casual analogies should be apparent" [54].

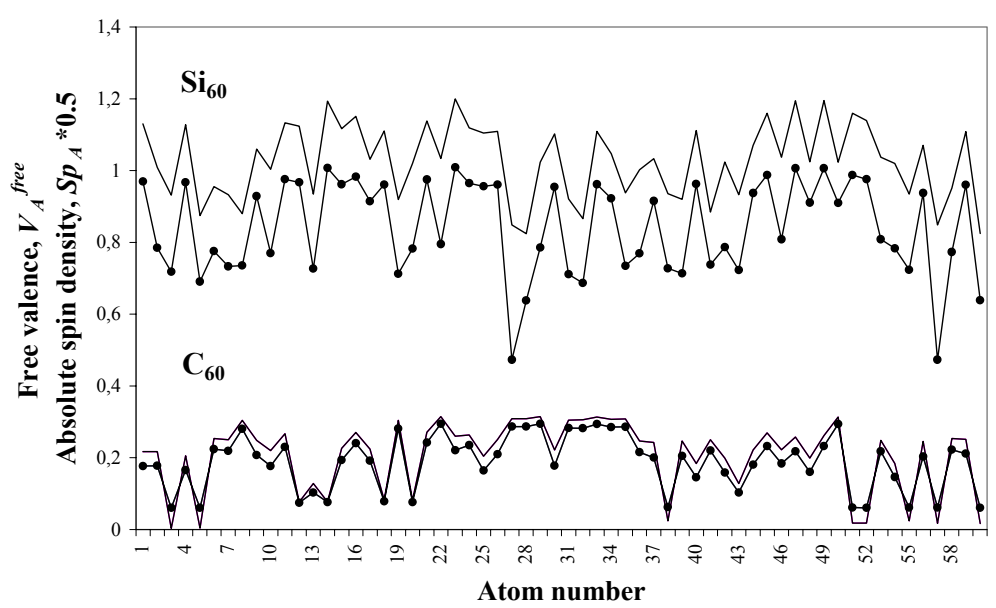

Figure 7. Free valence (solid curves) and absolute spin density (dotted curves) distributed over atoms of $\mathrm{C}_{60}$ and $\mathrm{Si}_{60}$ molecules 
Thus disclosed polyradical character of the basic fullerene molecule $\mathrm{C}_{60}$ is well consistent with extreme diversity of its properties which sometimes seem to be even contradictory. Enough to mention, for example, diamagnetism of a free molecule and pristine $\mathrm{C}_{60}$ crystals $[55,56]$ and a ferromagnetic behavior of either carpet-like polymerized crystalline species [57] or TDAE- $\mathrm{C}_{60}$ charge complex [58,59]. However, even Salem and Rowland have already mentioned [20] that diradical electronic structure is readily amenable to any external effects caused by either intramolecular chemical substitution or by intermolecular interaction. Actually, our recent studies have shown that above mentioned peculiarities in the magnetic behavior are tightly connected with changing the main polyradical characteristics $E_{r a d}, J$, and $D_{s p, A}$.

A peculiar influence of the molecule structure when keeping the chemical composition can be demonstrated for the $\mathrm{C}_{60}$ molecule in a shape of $4 \_1$ knot [60]. Figure 8 shows a starting and two equilibrated structures related to the RHF and UHF singlet states while Table 5 presents main characteristic of the electronic state.

As seen from the figure and table, both computational schemes results in quite similar molecular structures of $S_{4}$ symmetry with constant length C-C bonds, shorter in the case of the UHF approach. Therewith, the radical character of the molecule is clearly evident. The energy of radicalization is large and constitutes $286.51 \mathrm{kcal} / \mathrm{mol}$; similarly large is the UHF squared spin value. Spin density at atoms of $\sim \pm 1$ is quite uniformly distributed over atoms, pointing to $\sim 50 \%$ - radicalization of the molecule.

Another examples of "strange" behavior of the $\mathrm{C}_{60}$ admixtures such as effective inhibition of radical destruction of polystyrene [61] as well as sensitization of penetration through cell membranes [62] should be obviously analyzed from this viewpoint.

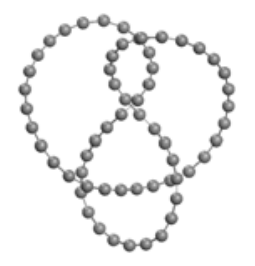

$C_{1}$

a



$S_{4}$

$\mathrm{b}$

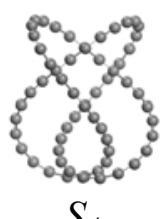

$S_{4}$

$\mathrm{c}$

Figure 8. Starting (a) and equilibrated structures of the $\mathrm{C}_{60} 4 \_1$ knot composition in the singlet RHF (b) and UHF (c) state. 
Table 5. Electronic characteristics of the $\mathrm{C}_{60} 4 \_1$ knot in the singlet state

\begin{tabular}{lcc}
\hline Quantity & $R H F$ & $U H F(S=0)$ \\
\hline & & \\
$\Delta \mathrm{H}, \mathrm{kcal} / \mathrm{mol}$ & 1961.45 & 1674.94 \\
$<\mathrm{C}-\mathrm{C}>, \mathrm{A}$ & 1.273 & 1.282 \\
Bond order & 1.825 & 1.633 \\
$\left(S^{* *} 2\right)$ & 0 & 18.13 \\
$D_{s p, A}$ & 0 & $+1.13 /-1.13$ \\
\hline
\end{tabular}

Polyradical character of the $\mathrm{Si}_{60}$ molecule is supported experimentally even more ponderably. The first consequence provided by the phenomenon may be formulated as a prohibition of the existence of particular chemical entities and even whole classes such as alkenes, alkynes, aromatic compounds, and fullerenes, typical for its carbon analogue. Only polyradicalization suppression can provide chemical stability that in the latter case may be realized in form of the $\mathrm{Si}_{60} \mathrm{H}_{60}$ and $\mathrm{Si}_{60} \mathrm{C}_{60}$ species $[9,10]$. This very high susceptibility of silicon atoms to polyradicalization provides as well magnetism of its crystal surfaces [63] that has already become a physical reality oppositely to the absence of similar effects for diamond crystal.

\section{Acknowledgements}

The author is greatly indebted to V.A.Zayets, M.V.Basilevsky, L.N.Shchegoleva for stimulating and valuable discussions and to D.Andrae for attracting the author attention to the $\mathrm{C}_{60}$ knot structures and supplying with coordinates of the starting 4 _ 1 configuration.

\section{References}

1. Chang, A.H.H.; Ermler, W.C.; Pitzer R.M.: J. Phys. Chem. 95, 1998, 9288.

2. Weaver, J.H. Acc. Chem. Res. 25, 1992, 143.

3. Bulusheva, L.G.: PhD Thesis, Institute of Inorganic Chemistry SB RAS, Novosibirsk 1998.

4. Lee, B.X.; Cao, P.L.; Que, D.L. Phys Rev 61B, $2000,1685$.

5. Nagase, S.: Pure Appl. Chem. 65, 1993, 675.

6. Slanina, Z.; Lee, S.L.: Fullerene Sci.Technol. 2, 1994, 459.

7. Lee, B.X.; Jiang, M.; Cao, P.L. J.Phys.: Condens. Matter 11, 1999, 8517.

8. Sheka, E.F.; Nikitina, E.A.; Zayets, V.A.; Ginzburg, I.Ya.: JETP Letters 71, 2001, 177.

9. Sheka, E.F.; Nikitina, E.A. Doklady RAN 378, 2001, 1. 
10. Sheka, E.F.; Nikitina, E.A.; Zayets, V.A.; Ginzburg, I.Ya. Int Journ Quant Chem 88, 2002,441 .

11. Kroto H.W., Heath J.R.; O’Brien S.C.; Curl R.F.; Smalley, R.E. Nature 318, 1985, 162.

12. Fowler, P.W.; Ceulemans, A. J.Phys.Chem. 99, 1995, 508.

13. It does not seem strange any more when comparing electron affinity of the fullerene $\mathrm{C}_{60}$ molecule $(2.66 \mathrm{eV})$ with those of aromatic molecules $(-0.55,-0.007,0.25,-2.45$, and $0.42 \mathrm{eV}$ for from benzene to pentacene, respectively). UHF calculated data by using AM1 technique (see text).

14. Herzberg, G.: Molecular Spectra and Molecular Structure. I.Spectra of Diatomic Molecules, $2^{\text {nd }}$ edn., Van Nostrand, Prinston, N.J. 1950, p.353.

15. Gubanov, V.A.; Likhtenstein, A.I.; Postnikov, A.V. Magnetism and Chemical Bonding in Crystals (in Russian), Nauka, Moskva 1985, Chapter 2.

16. Hay J.M.; Thomson, R.H. Organic Chemistry of Stable Free Radicals, Academic Pres, New York, 1955.

17. Hoffman, R.; Imamura, A.; Hehre, W.J. J.Amer.Chem.Soc. 90, 1968, 1499.

18. Hoffman, R. Chem.Commun. 240, 1969.

19. Hoffman, R. Accounts Chem.Res. 4, 1971, 1.

20. Salem, L.; Rowland, C. Angew.Chem. Intern Edit. 11, 1972, 92.

21. Hoffman, R. J.Chem.Phys. 39, 1963, 1397.

22. Hoffman, R. J.Chem.Phys. 40, 1964, 2474, 2480, and 2745.

23. Harrison, J.W. In W.Kirsme (Ed.): Carbene Chemistry, $2^{\text {nd }}$ edn., Academic Press, New York 1971, Chapter 5, p.159.

24. Ellis, D.E.; Freeman, A.J. J.Appl.Phys.39, 1968, 424.

25. Hay, P.J.; Thibeault, J.C.; Hoffman, R.: J Amer Chem Soc. 97, 1975, 4884.

26. Misurkin, I.A.; Ovchinnikov, A.A. Uspekhi Khimii 44, 1975, 393.

27. The configuration $\Psi_{4}$ is usually omitted since the relevant state energy is high.

28. Pople J.; Nesbet, R. J.Chem.Phys. 22, 1954, 571.

29. Löwdin, P.O. Phys Rev 97, 1955, 1509, 1513.

30. Paunz. R. Alternant Molecular Orbital Method, Philadelphia-London, 1967.

31. Noodleman, L. J Chem Phys 74, 1981, 5737.

32. Daul, C.A.; Ciofini, I.; Bencini, A. In K.D.Sen (Ed) Review of Modern Quantum Chemistry, World Scientific: Singapore, 2002, vol.2, 1247.

33. Heizenberg, W. Ztschr.Phys. 1928, 325.

34. Benard, M. J Chem Phys 71, 1979, 2546. 
35. Doubly occupied canonical molecular orbitals that describe paired electrons are omitted [31].

36. Mattheis, L.F. Phys.Rev. 123, 1961, 1219.

37. Zayets, V.A. CLUSTER-Z1: Quantum-Chemical Software for Calculations in the s,pBasis: Institute of Surface Chemistry, Nat. Ac.Sci. of Ukraine: Kiev, 1990.

38. Dewar, M.J.S.; Zoebisch, E.G.; Healey, E.F.; Stewart, J.J.P. J Amer Chem Soc 107, 1985, 3902.

39. Zhogolev, D.A.; Volkov, V.B. Methods, Algorithms and Programs for QuantumChemical Calculations of Molecules (in Russian), Naukova Dumka: Kiev, 1976.

40. Easy to show, that the ferromagnetic limit of $\left(\hat{S^{2}}\right)^{U H F}$ at $N_{\alpha}-N_{\beta}=2 n$, that corresponds to $S_{\max }=n$, is equal to $\left(\hat{S^{2}}\right)_{\text {max }}^{U H F}=1 / 4 N(N+2)=S_{\max }\left(S_{\max }+1\right)$.

41. Application of the above semi-empirical technique is not crucial for the study from a conceptual viewpoint. Only its highly effective computational facilities has favored the choice. Spin-polarized DFT techniques works in similar situations absolutely analogously $[42,43]$, however their rather modest computational efficiency seriously prevents from carrying out an extended computational experiment which involves multiple studying of large systems at different spin multiplicity.

42. Ginzberg, A.P. J Amer Chem Soc. 102, 1980, 111.

43. Norman, J.G.; Ryan, P.B.; Noodleman, L. J Amer Chem Soc. 102, 1980, 4279.

44. Hereinafter in the paper energetic parameters are presented by heats of formation, $\Delta H=E_{\text {tot }}-\sum_{A} E_{\text {elec }}^{A}+E H E A T^{A}$, where $E_{\text {tot }}=E_{\text {elec }}+E_{\text {nuc }} . E_{\text {elec }}$ and $E_{\text {nuc }}$ are electronic and nuclear energies of the studied system, $E_{\text {elec }}^{A}$ is the electronic energy of an isolated atom $A$ and $E H E A T^{A}$ is the heat of formation for atom $A$. All values are calculated within the same computational session.

45. Brief Chemical Encyclopedia (in Russian), Sov. enziklopedia:Moscow, 1963, p.799.

46. Bulychev, B.M.; Udod, I.A. Russian Chem. Journ. 39, No.2, 1995, 9.

47. Alekseev, N.I.; Dyuzhev,G.A. Techn.Phys. 46, 2001, 573, 577.

48. Tomilin, F.N.; Avramov, P.V.; Varganov, S.A.; Kuzubov, A.A.; Ovchinnikov, S.G. Phys.Sol.State, 43, 2001, 973.

49. The disclosed feature has forced us to check the tendency for a series of aromatic hydrocarbons. The calculations have convincingly shown the strengthening of the effect 
when going from naphthalene to pentacene for both carbon and silicon species. The results will be partially discussed in the next Section.

50. Khavryutchenko, V.; Sheka, E., Aono, M.; Huang, D.-H. Phys. Low-Dim. Struct. No.3/4, 1998, 81.

51. A generalized bond index is determined as $K_{A B}=\left|P_{A B}\right|^{2}+\left|D_{s p, A B}\right|^{2}[52]$, where the first term is known as Wiberg's bond index [53] which was introduced to characterize the covalence of chemical bonds in molecules with closed electron shells while the second goes from the spin density matrix.

52. Semenov, S.G. In Evolution of the Valence Doctrine (in Russian), Moscow, Khimia, 1977, p. 148.

53. Wiberg, K.B.Tetrahedron, 24, 1968, 1083.

54. Gaspar, P.; Herold, B.J. In W.Kirsme (Ed.): Carbene Chemistry, $2^{\text {nd }}$ edn., Academic Press, New York, 1971, Chapter 13, p.504.

55. Haddon, R.C.; Scheemeyer, L.F.; Waszczak, J.V.; Glarum, S.H., Tycko, R.; Dabbah, G.; Kortan, A.R.; Muller, A.J.; Mujsce, A.M.; Rosseinsky, M.J.; Zahurak, S.M.; Makhija, A.V.; Thiel, F.A.; Raghavachari, K.; Cockayne, E.; Elser V. Nature 350,1991, 46.

56. Luo, W.L.; Wang, H.; Ruoff, R.C.; Cioslowski, J.; Phelps, S. Phys. Rev. Lett. 73, 1994, 186.

57. Makarova, T.L.; Sundqvist, B.; Esquinazi, P.; Höhne, R.; Kopelevich, Y.; Scharff, P.; Davydov, V. A.; Kashevarova, L. S., Rakhmanina, A. V. Nature 413, 2001, 716.

58. Allemand P.-M.; Khemani K.C.; Koch A.; Wudl F.; Holczer K.; Donovan S.; Grüner G.; Thompson J.D. Science 253, 1991, 301.

59. Sheka, E.F. $6^{\text {th }}$ Session of the V.A.Fock School on Quantum and Computational Chemistry, V.Novgorod University, V.Novgorod, 2003, p.149.

60. Brodbeck, R.; Andrae, D. $6^{\text {th }}$ Session of the V.A.Fock School on Quantum and Computational Chemistry, V.Novgorod University, V.Novgorod, 2003, p.88.

61. Volkova, N.N.; Sumannen, E.V. $13^{\text {th }}$ Symposium on Modern Physical Chemistry, Institute of Chemical Physics Problems of RAS, Tuapse, Russia, 2001, p.122

62. Kotelnikova, R.A.; Bogdanov, G.N.; Romanova, V.S.; Parnes, Z.N. Mol. Mat. 11, 1998, 111.

63. Sheka, E.F.; Nikitina, E.A.; Zayats, V.A. Surface Science 2003 (in print). 